\title{
EVOLUTIONARY DIAGNOSTICS OF LBV SPECTRA AND SYSTEMS IN THE LMC
}

\author{
NOLAN R. WALBORN \\ Space Telescope Science Institute \\ 3700 San Martin Drive \\ Baltimore, Maryland 21218, USA
}

\begin{abstract}
The characteristics of the Ofpe/WN9 class in the LMC, including two newly discovered members, are reviewed; a strong relationship between this class and the LBVs is established by HDE 269858 = Radcliffe 127, currently in outburst with a spectrum identical to that of $S$ Doradus. The evidence for CNOcycle products, i.e., enhanced $\mathrm{N}$ and $\mathrm{He}$, on the surfaces and in the circumstellar nebulae of Ofpe/WN9 objects and LBVs is discussed, with emphasis on recent near-IR observations of He/H. Finally, the occurrence of several Ofpe/WN9 and LBV stars in compact multiple systems will enable valuable information about the evolutionary status of the peculiar objects to be derived. Strong threads unite these three topics: the same objects frequently recur in each.
\end{abstract}

\section{The Ofpe/WNg Class}

\subsection{DEFINITION AND MEMBERSHIP}

A group of four unusual, hot emission-line spectra was isolated among the brightest OB supergiants in the Large Magellanic Cloud by Walborn (1977); their outstanding peculiarity is the combination of high-ionization Of (He II, N III) and low-ionization (He I, N II) emission features with comparable strengths. Despite their overall similarity, when examined in detail they divide into three subgroups, particularly in terms of the rich spectral morphology in the vicinity of $\mathrm{H} \delta$ : HDE 269858 and Sanduleak (Sk) $-67^{\circ} 266$ show exceptionally narrow absorption lines of Si IV $\lambda 4089$ and N III $\lambda 4097$; HDE 269227 displays a striking configuration of partially overlapping P Cygni profiles; while HDE 269445 has no absorption features except for a possible diffuse N III $\lambda 4097$. 
Subsequent discoveries have expanded the membership of the class, but not the number of subgroups. Sk-66 ${ }^{\circ} 40$ (Walborn 1986) and HDE 269582 (Stahl 1986) belong to the HDE 269858/Sk-67 266 subclass; HDE 269927C (Azzopardi and Breysacher 1979) and Bohannan - Epps (BE) 381 (Bohannan 1979) are similar to HDE 269227 (see also Walborn 1982); while no further spectra exactly like that of HDE 269445 have been found to date. Very recently, two new examples of the HDE 269858/Sk $-67^{\circ} 266$ subclass have been identified by Bohannan and Walborn (1988); they are HDE 269687 and $\mathrm{Sk}-69^{\circ} 297$, which bring the membership of the Ofpe/WN9 class in the LMC to ten. This last reference also illustrates the spectra of most members of the class in terms of modern digital data, as a basis for future work.

There is some indication that the envelopes of the HDE 269227 subgroup have higher ionization than those of the HDE $269858 / \mathrm{Sk}-67^{\circ} 266$ type, in the sense that the He II/He I and N III/N II emission-line ratios are larger in the former, despite similar ranges in line strengths. The differing appearances near $\mathrm{H} \delta$ may then be due to relatively stronger Si IV and N III emissions there in the HDE 269227 subclass.

\subsection{VARIATIONS}

In or about 1980, the Ofpe/WN9 prototype HDE $269858=$ Radcliffe 127 unexpectedly entered an outburst phase (Stahl et al. 1983, Walborn 1984), during which its spectrum has evolved through an intermediate B-type to a peculiar supergiant A-type identical to that of S Doradus, while the bolometric luminosity has remained constant (Stahl and Wolf 1986a, Wolf et al. 1988). Thus HDE 269858 has firmly established its own LBV nature, as well as a connection between the Ofpe/WN9 and LBV classes. A connection in the opposite direction has been made by the recent discovery of Ofpe/WN9 characteristics during a light minimum of the galactic LBV AG Carinae (Stahl 1986), which at other times displays a B-type spectrum identical to that of $P$ Cygni (Hutsemékers and Kohoutek 1988), and an A-type similar to $S$ Doradus (Wolf and Stahl 1982).

These remarkable observations indicate previously unsuspected relationships among the apparently disparate objects in question. Moreover, strong hypotheses are suggested that all Ofpe/WN9 objects may be quiescent LBVs, and conversely that many or perhaps all LBVs are of Ofpe/WN9 type at minimum. A corollary of the latter would be that the true minimum states of $P$ Cygni and $S$ Doradus have not yet been observed, and that they are in extended intermediate or maximum states.

A different kind of variability is shown by HDE 269582, which evidently oscillates between high-ionization (Ofpe/WN9) and low-ionization (Bpe) states (Shore and Sanduleak 1984, Stahl 1986, Bohannan and Walborn 1988). The relation be- 
tween these spectrum variations and the poorly observed light variations of this object has not yet been established. The recent reappearance of its Ofpe/WN9 spectrum is delimited to sometime between November 1983 and December 1984 by observations shown by Stahl (1986) and Bohannan and Walborn (1988). This behavior also raises a question whether other similar Bpe objects in the LMC might be members of the Ofpe/WN9 class in transitory low-ionization disguise.

\section{Evolutionary Abundance Anomalies}

The evidence for He and CNO anomalies in Ofpe/WN9 and LBV objects was recently reviewed by Walborn (1988) in the context of such effects in a wider array of massive stellar atmospheres and winds, so that only the salient points and more recent developments will be summarized here.

\subsection{CIRCUMSTELLAR NEBULAE}

Another important property of several Ofpe/WN9 objects in the LMC, including HDE 26985, is their associated nitrogen-rich, nonspherical circumstellar nebulae, which were first detected spectroscopically by Walborn (1982). Stahl and Wolf (1986b) found evidence for similar structures in the spectra of a wider range of LMC emission-line supergiants, including the LBVs S Doradus and HDE 269006 $=\mathrm{R} 71$. In the case of the galactic LBV AG Carinae, a spectacular ring nebula can be observed directly in detail, and the first abundance results indicating nitrogen enhancement and oxygen depletion in this object have recently been reported by Dufour and Mitra (1987).

Eta Carinae may be regarded as the most extreme LBV. The large $\mathrm{N}, \mathrm{He}$ overabundances and $\mathrm{C}, \mathrm{O}$ underabundances in its spectacular ejected nebulosity have been discussed by Davidson, Walborn, and Gull (1982); Allen, Jones, and Hyland (1985); and Davidson, Dufour, Walborn, and Gull (1986).

\subsection{LBV B-TYPE SPECTRA}

The $\lambda 4600$ region in early-B supergiant spectra provides a sensitive diagnostic of CNO anomalies, as discussed by Walborn $(1971,1972,1976)$; when $N$ dominates over $\mathbf{C}$ and $\mathrm{O}$, striking departures from the normal line-strength patterns occur. In every well-observed LBV B-type phase, such dominance by $\mathrm{N}$ II is seen. The examples include P Cygni (Luud 1967), AG Carinae (Hutsemékers and Kohoutek 1988), AF Andromedae and M33 Variable B (Kenyon and Gallagher 1985), HDE $269006=$ R71 (Wolf, Appenzeller, and Stahl 1981), and HDE $269858=$ R127 (Stahl et al. 1983). No quantitative abundance analyses yet exist for such spectra, but it is virtually certain that when they are done large nitrogen overabundances will be found. 


\subsection{HE/H IN THE IR}

The unprecedented quality of the recent $2 \mu$ spectroscopic survey of LMC emissionline supergiants by McGregor, Hillier, and Hyland (1988 - see also these Proceedings) has led to several significant new results, including the identification of a category in which the $\mathrm{He} \mathrm{I} 2.058 \mu$ line is stronger than $\mathrm{H}$ Brackett- $\gamma$, which they have reasonably suggested to indicate an abundance anomaly. Of the eight objects in this category, five are members of the Ofpe/WN9 class (HDE 269445, 269582, 269687; Sk $-66^{\circ} 40,-67^{\circ} 266$ ), while the other three have been suggested to be related to it by Stahl and Wolf (1987) and Bohannan and Walborn (1988). This is a remarkable degree of a posteriori correlation! Evidently the morphological categories are well defined.

Two Ofpe/WN9 objects observed by McGregor et al. did not show He>H, but plausible explanations are available in both cases. One is HDE 269858 observed in its A-type outburst state, and the $2 \mu$ spectrum is also identical to that of $S$ Doradus; the $\mathrm{He} \mathrm{I}$ is most likely not seen because the ionization is too low, and it is predicted that if these objects can be observed at $2 \mu$ in Ofpe/WN9 states, they will show $\mathrm{He}>\mathrm{H}$. The other case is HDE 269227, in which a red supergiant companion dominates the $2 \mu$ region and also the ionization is higher than in the other observed objects as noted above. Moreover, from a quantitative analysis of the optical spectrum of HDE $269227=\mathrm{R} 84$, W. Schmutz, C. Leitherer et al. (these Proceedings) have in fact derived a substantial He overabundance, as well as a surprisingly low initial mass of $25 M_{\odot}$, a point which will recur in the next section.

All of these results provide strong evidence that both the Ofpe/WN9 class and the LBVs are highly evolved objects, which have CNO-cycle products at their surfaces and are ejecting them into their circumstellar environments.

\section{Multiple Systems}

A number of Ofpe/WN9 and LBV objects in the LMC are located in compact multiple systems which are most likely physically associated and coeval. The only case which has been investigated in detail so far is that of HDE 269227 by Schild (1987), who found that the associated stars are predominantly of type B and derived a likely initial mass for HDE 269227 of $25 M_{\odot}$-in perfect agreement with the independent determination from the atmospheric analysis by Schmutz et al. cited above. Other outstanding multiple systems about which little or nothing is known are those including HDE 269858; HDE 269927C (Azzopardi and Breysacher 1979, Walborn 1982); and S Doradus (Leitherer et al. 1985). Another spectacular case involving a different kind of emission-line supergiant is that of HDE 269599 (Appenzeller et al. 1984). Detailed spectroscopic and photometric 
studies of these systems will provide unique information about the initial mass, age, and evolutionary status of the peculiar objects.

\section{REFERENCES}

Allen, D.A., Jones, T.J., and Hyland, A.R. 1985, Ap. J., 291, 280.

Appenzeller, I., Klare, G., Stahl, O., Wolf, B., and Zickgraf, F.-J. 1984, ESO Messenger, No. 38, p.28.

Azzopardi, M., and Breysacher, J. 1979, Astr. Ap., 75, 243.

Bohannan, B.E. 1979, in IAU Symposium 83, Mass Loss and Evolution of O-Type Stars, ed. P.S. Conti and C.W.H. de Loore (Dordrecht: Reidel), p. 479.

Bohannan, B.E., and Walborn, N.R. 1988, in press.

Davidson, K., Dufour, R.J., Walborn, N.R., and Gull, T.R. 1986, Ap. J., 305, 867.

Davidson, K., Walborn, N.R., and Gull, T.R. 1982, Ap. J. (Letters), 254, L47.

Dufour, R.J., and Mitra, P. 1987, B.A.A.S., 19, 1090.

Hutsemékers, D., and Kohoutek, L. 1988, Astr. Ap. Suppl., 73, 217.

Kenyon, S.J., and Gallagher, J.S. III 1985, Ap. J., 290, 542.

Leitherer, C., Appenzeller, I., Klare, G., Lamers, H.J.G.L.M., Stahl, O., Waters, L.B.F.M., and Wolf, B. 1985, Astr. Ap., 153, 168.

Luud, L.S. 1967, Soviet Astr., $11,211$.

McGregor, P.J., Hillier, D.J., and Hyland, A.R. 1988, preprint.

Schild, H. 1987, Astr. Ap., 173, 405.

Shore, S.N., and Sanduleak, N. 1984, Ap. J. Suppl., 55, 1.

Stahl, O. 1986, Astr. Ap., 164, 321; err. 170, 197.

Stahl, O., and Wolf, B. 1986a, Astr. Ap., 154, 243. . 1986b, Astr. Ap., 158, 371. . 1987, Astr. Ap., 181, 293.

Stahl, O., Wolf, B., Klare, G., Cassatella, A., Krautter, J., Persi, P., and FerrariToniolo, M. 1983, Astr. Ap., 127, 49.

Walborn, N.R. 1971, Ap. J. (Letters), 164, L67. . 1972, Ap. J. (Letters), 176, L119.

. 1976, Ap. J., 205, 419. . 1977, Ap. J., 215, 53. . 1982, Ap. J., 256, 452. . 1984, In IAU Symposium 108, Structure and Evolution of the Magellanic Clouds, ed. S. van den Bergh and K.S. de Boer (Dordrecht: Reidel), p. 239. 
1986, in IAU Symposium 116, Luminous Stars and Associations in Galaxies, ed. C. W. H. de Loore, A. J. Willis, and P. Laskarides (Dordrecht: Reidel), p. 185. 1988, in IAU Colloquium 108, Atmospheric Diagnostics of Stellar Evolution, ed. K. Nomoto, Lecture Notes in Physics, 305 (Berlin: SpringerVerlag), p. 70.

Wolf, B., Appenzeller, I., and Stahl, O. 1981, Astr. Ap., 103, 94.

Wolf, B., and Stahl, O. 1982, Astr. Ap.,112, 111.

Wolf, B., Stahl, O., Smolinski, J., and Cassatella, A. 1988, Astr. Ap. Suppl., 74, 239.

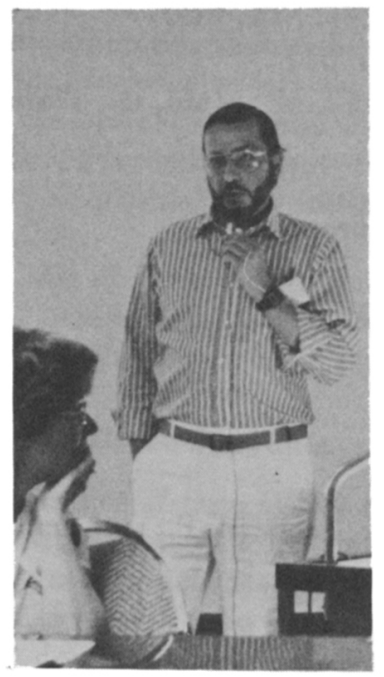

Nolan Walborn 


\section{DISCUSSION}

De Groot: A spectrum of P Cygni is sometimes classified as $\mathrm{OBN}$, Ofpe, Of/WN, or otherwise. Don't we have a problem of nomenclature?

Walborn: I would not classify P Cyg as any of those, only as B1 Iape. He II $\lambda 4686$ emission would be required for an Ofpe/WN9 classification, and this has never been seen in P Cyg so far as I know. Your interesting light curve shows that P Cyg has remained at an intermediate level since the 17 th century, consistent with its relatively constant intermediate-B-type LBV spectrum. If we could observe it spectroscopically at the extreme minimum and maximum levels as reached during the 17 th century, we might see Ofpe/WN9 and A-type spectra, respectively.

Heap: What is the difference between late OIaf spectra and Ofpe/WN spectra? What percentage of stars is in each class?

Walborn: The original defining characteristic was that the Ofpe/WN9 spectra show strong low-ionization emission features (N II, He I) along with the normal highionization Of-features (N III, He II). In the LMC, ten Ofpe/WN9 objects are now known, which is comparable to the number of normal of stars known there. However, the statistics are most likely incomplete; the situation in our Galaxy is much worse, though improving.

Maeder: For the interesting star HDE 269227 studied by Schmutz and by Schild, do you have any CNO-ratio data in addition to the helium abundance?

Walborn: No, the analysis includes only $\mathrm{He}$ and $\mathrm{H}$; but $\mathrm{I}$ believe the study of additional elements is planned.

Langer: Is any LBV known not to have CNO-cycled surface material? Such objects might exist, according to standard evolutionary calculations.

Walborn: None to my knowledge. All cases for which there is relevant evidence have nitrogen enhancements.

Kudritzki: Just related to your question, Norbert -- we have quantitatively studied nine B Ia -type supergiants (poster paper, this meeting) and none of them has a normal helium abundance. Interestingly, these stars are classified as apparently normal. Atmospheric CNO-cycle enrichment is more common in the post-mainsequence stage than previously thought.

Walborn: This point was addressed in my review at IAU Colloquium 108 in Tokyo. There is growing evidence that the morphologically normal majority of OB supergiants have CNO-cycle products in their atmospheres, while the rarer morphologically nitrogen-deficient ones (e.g. OBC) have normal (i.e. main sequence) abundances.

Sreenivasan: Is there any evidence of radial motion in the nebula around AG Carinae? Is there inward or outward motion?

Walborn: Thackeray found that it is expanding at about $50 \mathrm{~km} / \mathrm{s}$. Paresce (these proceedings) will show us evidence for more complex kinematics.

Conti: Line widths and expansion velocities of some of these stars imply relatively slow outflow, not all that different from that of supergiants.

Humphreys: Did you say that R 71 is a multiple system? If so this is new.

Walborn: No, this was a misunderstanding. $\mathrm{R} 71$ is not known to be multiple.

Leitherer: This was why we did our first fine-analysis of an LBV with $R 71$ and not with R 127 . Unfortunately $R 127$ has a close companion which may affect the observations. 
Wolf: I should like to note that S111 in the LMC has recently been found to be a B[e] star (see poster paper by Stahl et al.).

Shore: HD 38489 (=S 134/LMC) is a member of a "normal" populous blue cluster and its place in the H-R diagram is consistent with a high mass $\left(\sim 50 \mathrm{M}_{\odot}\right)$. HDE 269445 $(=\mathrm{S} \mathrm{30} / \mathrm{LMC})$ is one star with He II $\lambda 4686$ and may be helium-enhanced. It is nitrogen-rich and is one of the highest-excitation objects in the LMC sample.

Walborn: It is worth noting that you found nitrogen enhanced in HD 38489. 